Case Reports

\title{
COVID-19 Diagnosis in Asymptomatic: RT-qPCR or any other Cost-Effective Method?
}

\author{
${ }^{1}$ Mayank Agarwal, ${ }^{2}$ Yogesh Arvind Bahurupi and ${ }^{1}$ Prasan Kumar Panda \\ ${ }^{1}$ Department of Internal Medicine (Infectious Disease Division), All India Institute of Medical Sciences (AIIMS), \\ Rishikesh, Uttarakhand, India \\ ${ }^{2}$ Community and Family Medicine, All India Institute of Medical Sciences (AIIMS), Rishikesh, Uttarakhand, India
}

\begin{abstract}
Article history
Received: 02-03-2021

Revised: 18-05-2021

Accepted: 19-05-2021

Corresponding Author:

Prasan Kumar Panda

Department of Internal Medicine

(Infectious Disease Division), All

India Institute of Medical Sciences (AIIMS), Rishikesh, Uttarakhand, India

Email: motherprasanna@ rediffmail.com
\end{abstract}

\begin{abstract}
On January $30^{\text {th }}$, 2020, the World Health Organization announced the COVID-19 outbreak as a Public Health Emergency of International Concern. In the view of this pandemic, early diagnosis is the mainstay for halting the disease progression. Quantitative real-time Reverse Transcriptase-Polymerase Chain Reaction (RT-qPCR) has been established as the cornerstone for the diagnosis of COVID-19. However, the significance of RT-qPCR positivity in asymptomatic cases with travel history, mass screening purposes, or close contact tracing remains debatable as their period of infectivity is unknown. We present a case series of 42 asymptomatic patients, who tested positive for COVID-19 and were subjected to hospitalization until they tested negative as per Government guidelines. Through our case series, we have tried to establish that RT-qPCR testing as a diagnostic criterion for asymptomatic patients with no known contact history can lead to increased psychological and economic burden on the Government, the patient as well as his family. It also overburdens the health care resources and therefore, raises the question about its necessity among this cohort of asymptomatic cases and thus the possible role of other methods in the diagnosis and isolation of such cases.
\end{abstract}

\section{Keywords: RT-PCR, COVID-19}

\section{Introduction}

In late 2019, a novel coronavirus, designated as SARSCoV-2, was identified as the cause of an outbreak of acute respiratory illness in Wuhan, a city in Hubei, China. Later, it was renamed 'COVID-19' by the World Health Organization (WHO) and by March 2020 the outbreak was characterised as a pandemic (Güner et al., 2020). Quantitative real-time Reverse Transcriptase-Polymerase Chain Reaction (RT-qPCR) is the mainstay for the diagnosis of COVID-19 (Wang et al., 2020). However, the detection of viral RNA does not necessarily indicate the presence of an infectious or viable virus. In a local study from a multi-center cohort of 73 COVID-19 patients in Singapore, when the cycle threshold value was higher (i.e., when the viral load was low), no viable virus (based on being able to culture the virus) was found. In addition, the virus could not be isolated or cultured after day 11 of the illness. This indicated that while viral RNA detection may persist in some patients, such persistent RNA detection represents a non-viable virus and such patients are non-infectious. Therefore, the current criteria for hospital discharge or discontinuation of quarantine need to be re-evaluated (NCIDS, 2020).

The same issue continues for asymptomatic cases who are tested RT-qPCR positive either after travel history, for mass screening purposes, or as part of close contact tracing. Once declared positive, there is fear, stress, hype and chaos at the individual, family and society levels. Hence, we should be sure what this positivity means and if we cannot decide the infectivity of such patients, then why are we testing them with qRT-PCR?

Therefore, we present a retrospective case series of all asymptomatic patients who tested positive in a hospital and decided on the requirement of RT-qPCR testing in the same cohort.

\section{Case Series}

\section{Study Design and Setting}

The present case series was conducted in a tertiary health care centre in North India. The study was approved by the institutional ethics committee for data collection, retrospectively from April to June 2020 (CTRI/2020/08/027169).

\section{Study Participants and Data Collection}

All patients were screened using the COVID-19 Screening OPD. Both nasal and oropharyngeal swabs were collected and sent to the laboratory in Viral Transport Medium (VTM). "SD Biosensor standard M nCoV real-time 
detection kit" was used for PCR for all patients. We used a Bio-Rad CFX 96 real-time thermocycler" and a ThermoFlex 96 extractor machine" for RNA extraction. The target genes were "E-gene and RdRp". According to the Indian Council of Medical Research (ICMR) guideline, a Cycle Threshold (CT) value of less than 35 was considered positive, whereas a value greater than 35 was considered negative. The first 100 RT-qPCR positive COVID-19 patients, admitted to the isolation ward of a tertiary care hospital, were enrolled in this study. Of these, 42 patients who fulfilled the following inclusion criteria were included in the study.

Inclusion criteria: All asymptomatic RT-qPCR positive COVID-19 cases with:

1. Travel history from outside the area of residence or

2. History of high-risk exposure with confirmed COVID-19 case

3. No documented travel or contact history

Exclusion criteria:

\section{All symptomatic COVID-19 cases}

Data were collected from electronic medical patient records retrospectively for demographic details, employment details in context of hospital, contact or travel history and hospitalisation details, including the date of admission and the duration of hospitalisation.

\section{Statistical Analysis}

Categorical variables were described as frequencies and percentages and continuous variables were described using mean and range. All data entries and statistical analyses were performed using Microsoft Excel 2019.

\section{Results}

Out of the first 100 RT-qPCR positive patients admitted, a total of 42 patients were asymptomatic, of which the majority were males and from outside hospital settings (not employed as health care workers) with a mean age of $32.9 \pm 14.6$ years with a range of 1 to 75 years (Table 1$)$. The mean duration of hospitalisation was $25.5 \pm 9.2$ days.

Ten patients reported having a contact history with a positive case and 19 had a travel history from interstate or intrastate hotspot areas. Eight patients had no documented travel or contact history, while five reported having both contact and travel history (Fig. 1).

According to the government COVID-19 testing strategies, all symptomatic and asymptomatic patients with a positive contact history/travel history were recommended to undergo RT-qPCR. On testing positive, all cases were quarantined in COVID-19 dedicated centres or hospitalised. Hence, all asymptomatic patients were admitted as per their RT-qPCR report and their duration of stay was assessed until they were discharged once negative.

Table 1: Distribution of asymptomatic RT-qPCR positive COVID-19 patients ( $\mathrm{N}=42)$

\begin{tabular}{lll}
\hline Variable & Sub-categories & Frequency $(\mathrm{N}, \%)$ \\
\hline Age Group & $<30$ & $20(47.6)$ \\
& $\geq 30$ & $22(52.4)$ \\
Sex & Male & $25(59.5)$ \\
Employment as a health care worker & Female & $17(40.5)$ \\
& Yes & $7(16.7)$ \\
Days of hospitalization & No & $35(83.3)$ \\
& $\leq 14$ & $8(19.0)$ \\
\hline
\end{tabular}

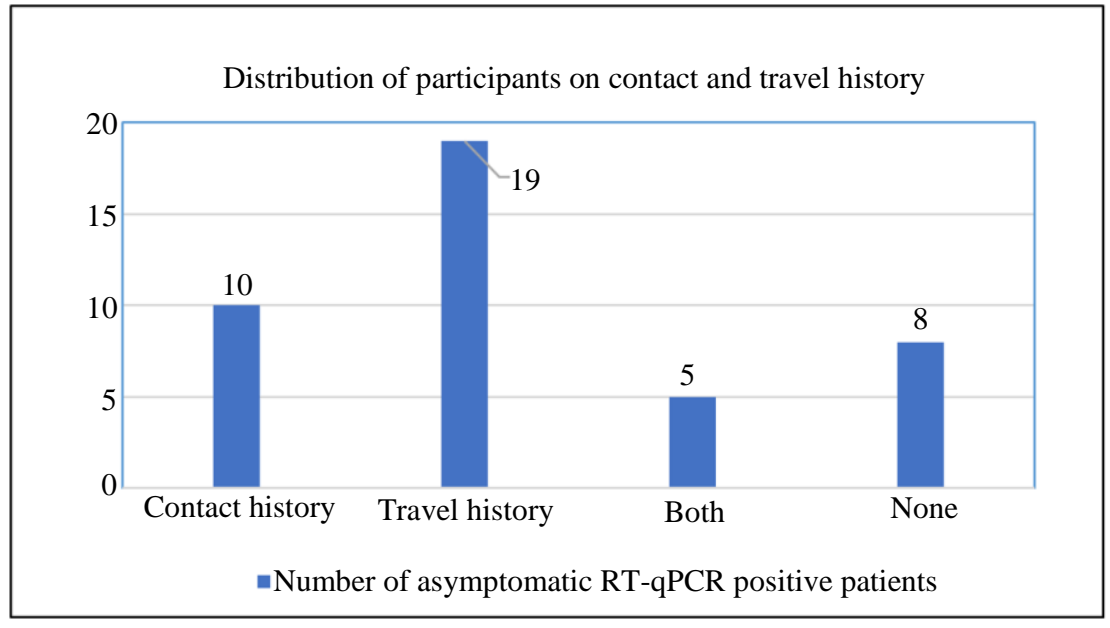

Fig. 1: Distribution of first asymptomatic RT-qPCR positive COVID-19 patients according to their contact and travel history ( $\mathrm{N}=42$ ) 


\section{Discussion}

Early COVID-19 detection holds the key to prevent a further rise in suspected or confirmed cases through community transmission and halt disease progression. In the present case series, all patients who tested positive on RT-qPCR testing were hospitalised for further treatment. Of the 42, 27 patients had a travel history or no documented records of contact history. This raises the question of whether all asymptomatic cases should undergo RT-qPCR testing leading to admission to a tertiary care centre due to the lack of a nearby COVID-19 care centre.

A recent study reported a positive retest in an asymptomatic, discharged and possibly non-infectious patient when tested by RT-qPCR for SARS-CoV-2 (Zhang et al., 2020). Similar results were observed in a study in China, where four patients with COVID-19 who met the criteria for hospital discharge or discontinuation of quarantine (absence of clinical symptoms and radiological abnormalities and two negative RT-qPCR test results) had positive RT-qPCR test results 5 to 13 days later (Lan et al., 2020). In another study, results from 70 COVID19 patients were analysed: 21 patients $(30 \%)$ had a positive third-time RT-qPCR test, even though the results were negative for the previous two tests. This suggests a high false-positive rate of RT-qPCR and questioned the criteria for discharge and discontinuation of quarantine (Xiao et al., 2020). In a recent publication, more emphasis was given on the cycle threshold value of RT-qPCR rather than positivity for de-isolation purposes (Kapoor et al., 2021).

Hence, relying on RT-qPCR test positivity for diagnosing and de-isolating asymptomatic patients cannot be very reassuring in the near future. This results in an unnecessary risk of exposure when admitted to a hospital, increasing their psychological distress and social stigma toward the disease in society. Moreover, in our study, the mean number of days of hospitalisation for asymptomatic patients was approximately 25 days. Based on the available data, the infectious period of SARS-CoV-2 in symptomatic individuals may begin approximately 2 days before the onset of symptoms and persist for approximately 7-10 days after the onset of symptoms. Active viral replication dropped quickly after the first week and a viable virus was not found after the second week of illness despite the persistence of RT-qPCR detection of RNA (NCIDS, 2020). Prolonged hospital stay in asymptomatic cases could be over burdened by the health care system in terms of overutilization of already limited hospital resources, including qRT-PCR kits, manpower, hospital beds, drugs, or funds. It also increases the economic burden on the patient and his family. Hence, we question the cost-effectiveness of qRT-PCR in such cases. Therefore, as per our case series, we suggest that the 27 asymptomatic patients with a travel history or no documented records of contact history could have been subjected to other cheaper diagnostic methods instead of RT-qPCR tests, leading to the conservation of resources. Even better would be to quarantine them without any testing and observe for 7days for any development of symptoms. With the progression of the pandemic, this has ultimately occurred nowadays, but the initial period of the pandemic was not prepared or foreseen to do the same resulting wastage of many resources.

In the present scenario of the COVID-19 pandemic, serological analysis may help to examine exposure, especially in asymptomatic cases. Serological testing is based on an enzyme-linked immunosorbent assay to detect IgM and IgG antibodies against the N-protein of SARS$\mathrm{CoV}-2$. A study calculated the sensitivity and specificity of serological assays of 77.3 and $100 \%$, respectively, for IgM and 83.3 and $95 \%$ for IgG (Xiang et al., 2020). Compared to RT-qPCR, serological assays have several advantages, such as rapid application, quicker results and detection of past infection (Stowell and Guarner, 2020).

In a recent review article, alternative molecular methods of diagnosis, such as Loop-Mediated isothermal Amplification (LAMP), have been suggested as it is a less costly and simpler procedure. They also focused on various antigen and antibody-based assays as point-ofcare tests for providing rapid diagnosis, especially in emergencies, mass screening purposes and even at the bedside (Alpdagtas et al., 2020).

Even though RT-qPCR continues to remain the gold standard test, we questioned its utility in asymptomatic cases. More research is required to consider alternative diagnostic tests with better cost effectiveness when applied for screening large populations, which will allow rapid identification and isolation of infected patients. In addition, there is a need for a long-term strategy to prevent recurrent outbreaks of infection, which would imply repeated and regular mass testing.

Thus, home quarantine is a possible solution. If RTqPCR is really needed in asymptomatic patients, then specific categories should be defined, such as patients with immunosuppression.

\section{Conclusion}

Even though RT-qPCR continues to be the cornerstone for early detection of SARS-CoV-2, it could not answer in most asymptomatic cases since RT-qPCR positivity may be there for longer periods, even if the individual is not infectious. More importantly, asymptomatic patients are quarantined based on RT-qPCR positivity without knowing when infection enters the body, when known primary exposure is absent, leading to a burden on resources as well the patient, especially their physical and mental well-beings. The role of alternative strategies may be there for diagnosis and quarantine of such asymptomatic cases, but this needs further consideration. 
This will also lead to better utilisation of resources and timely public health interventions.

\section{Acknowledgment}

A gratitude to COVID-19 management team during this pandemic crisis.

\section{Author's Contributions}

Mayank Agarwal: Did literature search, collected data, drafted the manuscript and approved.

Yogesh Arvind Bahurupi: Did literature search, analysis data, reviewed the manuscript and approved.

Prasan Kumar Panda: Gave concept and design, interpreted data, reviewed critically the manuscript and approved.

\section{Ethics}

This article is original and contains unpublished material. The corresponding author confirms that all of the other authors have read and approved the manuscript and no ethical issues involved.

\section{References}

Alpdagtas, S., Ilhan, E., Uysal, E., Sengor, M., Ustundag, C. B., \& Gunduz, O. (2020). Evaluation of current diagnostic methods for COVID-19. APL bioengineering, $\quad 4(4), \quad 041506$. https://aip.scitation.org/doi/full/10.1063/5.0021554

Güner, H. R., Hasanoğlu, I., \& Aktaş, F. (2020). COVID-19: Prevention and control measures in community. TurkishJournal of MedicalSciences, 50(SI-1), 571-577. https://pubmed.ncbi.nlm.nih.gov/32293835/

Kapoor, M., Kalita, D., \& Panda, P. K. (2021). Cycle threshold values versus reverse transcriptionpolymerase chain reaction positivity in COVID-19 de-isolation. Indian Journal of Medical Microbiology, 39(1), 133-135.

https://pubmed.ncbi.nlm.nih.gov/33610247/
Lan, L., Xu, D., Ye, G., Xia, C., Wang, S., Li, Y., \& $\mathrm{Xu}$, H. (2020). Positive RT-PCR test results in patients recovered from COVID-19. JAMA, 323(15), 1502-1503.

http://www.nhc.gov.cn/yzygj/s7653p/202002/

NCIDS. (2020). Period of Infectivity to Inform Strategies for De-isolation for COVID-19 Patients. National Centre for Infectious Diseases Singapore. Natl Cent Infection Dis Chapter Infection Dis Physicians, 2020, 1-5.

Stowell, S. R., \& Guarner, J. (2020). Role of Serology in the Coronavirus Disease 2019 Pandemic [Internet]. Clinical Infectious Diseases. Oxford University Press, p, 1935-1936. https://www.ncbi.nlm.nih.gov/pmc/articles/PMC7 197618/

Wang, Y., Kang, H., Liu, X., \& Tong, Z. (2020). Combination of RT-qPCR testing and clinical features for diagnosis of COVID-19 facilitates management of SARS-CoV-2 outbreak. Journal of Medical Virology, 92(6), 538-539. https://pubmed.ncbi.nlm.nih.gov/32096564/

Xiang, F., Wang, X., He, X., Peng, Z., Yang, B., Zhang, J., ... \& Ma, W. L. (2020). Antibody detection and dynamic characteristics in patients with coronavirus disease 2019. Clinical Infectious Diseases, 71(8), 1930-1934. https://doi.org/10.1093/cid/ciaa461

Xiao, A. T., Tong, Y. X., \& Zhang, S. (2020). False negative of RT-PCR and prolonged nucleic acid conversion in COVID-19: Rather than recurrence. Journal of Medical Virology, 92(10), 1755-1756. https://pubmed.ncbi.nlm.nih.gov/32270882/

Zhang, J. F., Yan, K., Ye, H. H., Lin, J., Zheng, J. J., \& Cai, T. (2020). SARS-CoV-2 turned positive in a discharged patient with COVID-19 arouses concern regarding the present standards for discharge. International Journal of Infectious Diseases, 97, 212-214. https://pubmed.ncbi.nlm.nih.gov/32200109/ 